\title{
CONJUGATE POINTS, TRIANGULAR MATRICES, AND RICCATI EQUATIONS $\left({ }^{1}\right)$
}

\author{
BY
}

\section{ZEEV NEHARI}

\begin{abstract}
Let $A$ be a real continuous $n \times n$ matrix on an interval $\Gamma$, and let the $n$-vector $x$ be a solution of the differential equation $x^{\prime}=$ $A x$ on $\Gamma$. If $[\alpha, \beta] \in \Gamma, \beta$ is called a conjugate point of $\alpha$ if the equation has a nontrivial solution vector $x=\left(x_{1}, \ldots, x_{n}\right)$ such that $x_{1}(\alpha)=\ldots=x_{k}(\alpha)=$ $x_{k+1}(\beta)=\ldots=x_{n}(\beta)=0$ for some $k \in[1, n-1]$.

It is shown that the absence on $\left(t_{1}, t_{2}\right)$ of a point conjugate to $t_{1}$ with respect to the equation $x^{\prime}=A x$ is equivalent to the existence on $\left(t_{1}, t_{2}\right)$ of a continuous matrix solution $L$ of the nonlinear differential equation $L^{\prime}=\left[L A^{*} L^{-1}\right]_{\tau_{0}} L$ with the initial condition $L\left(t_{1}\right)=I$, where $[B]_{\tau_{0}}$ denotes the matrix obtained from the $n \times n$ matrix $B$ by replacing the elements on and above the main diagonal by zeros. This nonlinear equation-which may be regarded as a generalization of the Riccati equation, to which it reduces for $n=2-$ can be used to derive criteria for the presence or absence of conjugate points on a given interval.
\end{abstract}

Let $A=A(t)$ be a continuous real-valued $n \times n$ matrix on a real interval $\Gamma$. We consider the differential equation

$$
x^{\prime}=A x,
$$

whose solutions are $n$-dimensional vector functions $x(t)$, and the associated equation

$$
X^{\prime}=A X,
$$

whose solutions are $n \times n$ matrices $X(t)$. In both cases we shall consider only real solutions. If $t_{1}, t_{2} \in \Gamma$, the point $t_{2}$ will be said to be a (right) conjugate point of $t_{1}$ with respect to equation (1) or (2) if (1) has a nontrivial solution vector $x=\left(x_{1}, \cdots, x_{n}\right)$ such that, for some $k \in[1, n-1], x_{1}\left(t_{1}\right)$ $=\cdots=x_{k}\left(t_{1}\right)=x_{k+1}\left(t_{2}\right)=\cdots=x_{n}\left(t_{2}\right)=0$. A similar definition can be given for a left conjugate point, but it will not be necessary for our purposes, and we shall therefore use the term "conjugate point" to refer to the right conjugate point just defined.

We quote here some well-known elementary facts concerning equations

Received by the editors June 1, 1973 and, in revised form, December 4, 1973. AMS (MOS) subject classifications (1970). Primary 34C10; Secondary 34A30.

(1) Research supported by the National Science Foundation under grant GP23113. 
(1) and (2). A solution matrix $X$ of (2) which is nonsingular at one point of $\Gamma$ is nonsingular for all $t \in \Gamma$; such a solution is called a fundamental solution of (2). If $X$ is fundamental, all other solutions of (2) are of the form $X C$, where $C$ is a constant matrix. The general solution of the vector-matrix equation (1) is of the form $x=X \alpha$, where $X$ is a fundamental solution of (2) and $\alpha$ is a constant vector of dimension $n$.

Our principal concern is the derivation of criteria for the presence or absence of conjugate points associated with equations of the form (1) or (2). We shall find that in the consideration of these questions a basic role is played by the class of nonsingular lower triangular matrices, i.e., matrices $\left(s_{k l}\right)_{k, l=1, \ldots, n}$ such that $s_{k k} \neq 0$ and $s_{k l}=0$ if $k<l$. The class of these matrices will be denoted by $\tau$. To indicate that a matrix $L$ is nonsingular and upper triangular, we write either $L^{*} \in \tau$ (where $L^{*}$ is the transpose of $L$ ) or $L \in \tau^{*}$. The symbol $\tau_{0}$ will be used for the class of lower triangular matrices whose main diagonal terms are all zero. The "lower triangular part" of a general matrix $S$ (i.e., the matrix obtained from $S$ if the terms $s_{k l}(k<l)$ are replaced by zeros) will be denoted by $[S]_{\tau}$. If, in addition, the elements $s_{k k}$ are replaced by zeros, we obtain the matrix $[S]_{\tau_{0}}$.

We shall use the symbol $N_{k}(1 \leqslant k \leqslant n-1)$ for the class of vectors $\alpha=\left(\alpha_{1}, \cdots, \alpha_{n}\right)$ for which $\alpha_{1}=\alpha_{2}=\cdots=\alpha_{k}=0$, and the symbol $N_{k}^{\prime}$ for the class of vectors $\alpha$ with $\alpha_{n-k+1}=\alpha_{n-k+2}=\cdots=\alpha_{n}=0$. Clearly, $S \alpha \in N_{k}$ if $\alpha \in N_{k}$ and $S \in \tau$; similarly, $S^{*} \alpha \in N_{k}^{\prime}$ is a consequence of $\alpha \in N_{k}^{\prime}$ and $S \in \tau$. We also note that if, for any $k \in[1, n-1], \alpha \in N_{k}$ and $\alpha \in N_{n-k}^{\prime}$, $\alpha$ is necessarily the zero vector.

We now state our basic lemmas.

Lemma I. The point $t_{2}\left(t_{1}<t_{2}\right)$ is not a conjugate point of $t_{1}$ with respect to equation (1) if and only if there exists a solution $X$ of (2) such that $X\left(t_{1}\right) \in \tau$ and $X\left(t_{2}\right) \in \tau^{*}$.

LEMMA II. The interval $\left(t_{1}, t_{3}\right)$ does not contain a conjugate point of $t_{1}$ with respect to equation (1) if and only if (2) has a solution matrix of the form

$$
X=L^{*} S, \quad L, S \in \tau,
$$

where the matrices $L, S$ are differentiable on $\left(t_{1}, t_{3}\right)$, and $L^{*}\left(t_{1}\right)=I$ (the unit matrix).

We first prove the "if" part of these statements. Suppose $t_{2}$ is conjugate to $t_{1}$, i.e., suppose there exists a nontrivial solution $x$ of (1) such that, for some $k \in[1, n-1], x\left(t_{1}\right) \in N_{k}$ and $x\left(t_{2}\right) \in N_{n-k}^{\prime}$. Since $x=X \alpha$, where $X$ is a fundamental solution of (2) and $\alpha$ is a constant vector, we have $X^{-1}\left(t_{1}\right) x\left(t_{1}\right)=X^{-1}\left(t_{2}\right) x\left(t_{2}\right)$. We apply this to the solution $X$ described 
in Lemma I (which is fundamental because of $X\left(t_{1}\right) \in \tau$ ) and note that. under the assumptions of Lemma $\mathrm{I}, X^{-1}\left(t_{1}\right) \in \tau, X^{-1}\left(t_{2}\right) \in \tau^{*}$. Since $X^{-1}\left(t_{1}\right) x\left(t_{1}\right)$ $\in N_{k}$ and $X^{-1}\left(t_{2}\right) x\left(t_{2}\right) \in N_{n-k}^{\prime}$, it follows that $X^{-1}\left(t_{1}\right) x\left(t_{1}\right)$ must be the zero vector. But this implies $x\left(t_{1}\right)=0$, i.e., $x$ is the trivial solution $x=0$, contrary to our assumption. The conditions on $X$ in Lemma $\mathrm{I}$ are thus sufficient to prevent $t_{2}$ from being a conjugate point of $t_{1}$.

The corresponding assertion in Lemma II is an immediate consequence of this. If $t_{2}$ is a point in $\left(t_{1}, t_{3}\right)$, we replace $X$ by the fundamental solution $Y=X S^{-1}\left(t_{2}\right)$ and note that, by (3) and the assumption $L^{*}\left(t_{1}\right)=I, Y\left(t_{1}\right) \in$ $\tau$ and $Y\left(t_{2}\right) \in \tau^{*}$. Thus, by Lemma $I, t_{2}$ cannot be conjugate to $t_{1}$.

To prove the "only if" part in Lemma I, suppose that $t_{2}$ is not conjugate to $t_{1}$, and let $x$ be a nontrivial solution of (1) for which $x\left(t_{1}\right) \in N_{k}$, where $k$ may be any integer in $[1, n-1]$. If $X$ is a fundamental solution of (2) for which $x\left(t_{1}\right) \in \tau$, we then have $X^{-1}\left(t_{1}\right) x\left(t_{1}\right) \in N_{k}$ and therefore, because of $X^{-1}(t) x(t)=\alpha=$ const, $x\left(t_{2}\right)=X\left(t_{2}\right) \alpha$, where $\alpha \in N_{k}$ and the components $\alpha_{k+1}, \alpha_{k+2}, \cdots, \alpha_{n}$ of $\alpha$ may be given arbitrary values by imposing suitable unitial conditions on the components $x_{k+1}, x_{k+1}, \cdots$, $x_{n}$ of $x$. We assert that the $(n-k) \times(n-k)$ submatrix of $X\left(t_{2}\right)$ in the lower right-hand corner must be nonsingular. If this were not true, there would exist a nontrivial set $\alpha_{k+1}, \cdots, \alpha_{n}$ such that the last $n-k$ components of $x\left(t_{2}\right)=X\left(t_{2}\right) \alpha$ are all zero, i.e., $t_{2}$ would be conjugate to $t_{1}$, contrary to our assumption. Since $k$ may be any integer in $[1, n-1]$, this argument shows that if $t_{2}$ is not conjugate to $t_{1}$ all the square submatrices of $X\left(t_{2}\right)$ which contain the element in the lower right-hand corner of $X\left(t_{2}\right)$ must be nonsingular. By a well-known result (e.g. $\left[1\right.$, p. 35]), this implies that $X\left(t_{2}\right)$ may be written in the form $L^{*} S$, where $L, S \in \tau$.

Since $Y(t)=X(t) S^{-1}$ is a solution of (2) for which $Y\left(t_{1}\right) \in \tau$ and $Y\left(t_{2}\right) \in \tau^{*}$, this completes the proof of Lemma I. To show that the decomposition $X(t)=L^{*}(t) S(t)$ (where $t$ may now be identified with any point in $\left.\left(t_{1}, t_{3}\right)\right)$ also leads to the proof of Lemma II, we note that this decomposition can be made unique by the requirement that all the elements in the main diagonal of $L(t)$ be equal to 1 . If this is done, the elements of $L(t)$ and $S(t)$ can be expressed rationally in terms of the elements of $X(t)$ [1, p. 38]. Since the denominators appearing in these expressions are the determinants $\left|X_{\nu \mu}\right|_{\nu, \mu=k+1, \ldots, n}(k=1, \cdots, n-1)$, which were just shown not to vanish on $\left(t_{1}, t_{3}\right)$, the differentiability of the elements of $L$ and $S$ follows from that of the elements of $X$. This concludes the proof of Lemma II.

2. Our next result shows that the absence, on an interval $\left(t_{1}, t_{2}\right)$, of a point conjugate to $t_{1}$ with respect to equation (1) is equivalent to the fact that a certain nonlinear differential equation has a continuous solution on 
$\left[t_{1}, t_{2}\right)$. The statement of the theorem uses the symbol $[\cdot]_{\tau_{0}}$ which, as indicated above, is defined as follows: If $B$ is a matrix $\left(b_{k m}\right)_{k, m=1, \ldots, n}$, then $C=[B]_{\tau_{0}}$ is the matrix $\left(c_{k m}\right)$ for which $c_{k m}=b_{k m}$ for $m<k$ and $c_{k m}=0$ for $m \geqslant k$.

THEOREM I. In order that the interval $\left(t_{1}, t_{2}\right)$ contain no point conjugate to $t_{1}$ with respect to equation (1), it is necessary and sufficient that the solution of the nonlinear differential equation

$$
L^{\prime}=\left[L A^{*} L^{-1}\right]_{\tau_{0}} L
$$

with the initial condition $L\left(t_{1}\right)=I$ be continuous on $\left(t_{1}, t_{2}\right)$.

We note that, if it exists, a continuous solution of (4) is necessarily of the form $L=I+L_{0}$, where $L_{0} \in \tau_{0}$. Indeed, since $L$ is a solution of the linear equation $L^{\prime}=R L$ where the matrix $R=\left[L A^{*} L^{-1}\right]_{\tau_{0}}$ is in $\tau_{0}$, we have $L=\lim _{m \rightarrow \infty} L_{m}$, where

$$
L_{m+1}=I+\int_{t_{1}}^{t} R L_{n} d s, L_{1}=I .
$$

Since $R L_{m} \in \tau_{0}$ if $R \in \tau_{0}$ and $L_{m} \in \tau$, this shows that $L_{m+1}-I \in \tau_{0}$ for all $m$, and the assertion follows.

Accordingly, the matrix $L$ has only $1 / 2 n(n-1)$ nontrivial elements, and equation (1) may therefore be tested for the absence of a conjugate point to $t_{1}$ in an interval $\left(t_{1}, t_{2}\right)$ by solving a system of $1 / 2 n(n-1)$ nonlinear differential equations. For $n=2$, only one equation has to be solved. As we shall see, this is precisely the classical Riccati equation associated with the $2 \times 2$ system of linear equations. The system (4) may thus be regarded as a generalization of the Riccati equation to the case of an $n \times n$ matrix. The nature of the nonlinearities appearing in (4) is described in the following statement.

If $L_{i k}(i>k)$ are the nontrivial elements of the solution matrix $L$ in (4), equation (4) is equivalent to a system

$$
L_{i k}^{\prime}=F_{i k}\left(L_{r s}\right) \text {, }
$$

where the $F_{i k}$ are polynomials of degree not higher than $n$ in the variables $L_{r s}(r>s)$.

To prove Theorem I we note that by Lemma II the absence of a conjugate point to $t_{1}$ in $\left(t_{1}, t_{2}\right)$ is equivalent to the existence on $\left[t_{1}, t_{2}\right)$ of a solution $X$ of (2) which admits of a decomposition $X=L^{*} S$, where $L, S \in \tau$, $L\left(t_{1}\right)=I$, and $L, S$ are differentiable on $\left[t_{1}, t_{2}\right)$. As pointed out above, we may also assume that, throughout $\left[t_{1}, t_{2}\right)$, all the elements in the main diagonal of $L$ are equal to 1 . Substituting this representation of $X$ in (2), we have 
$L^{* \prime} S+L^{*} S^{\prime}=A L^{*} S$, and thus $L^{*-1} L^{* \prime}+S^{\prime} S^{-1}=L^{*-1} A L^{*}$ or, equivalently,

$$
L^{\prime} L^{-1}+S^{*-1} S^{* \prime}=L A^{*} L^{-1} .
$$

Since $S \in \tau$, we have $S^{*-1} S^{* \prime} \in \tau^{*}$ and therefore $\left[S^{*-1} S^{* \prime}\right]_{\tau_{0}}=0$. From $L-I \in \tau_{0}$ it follows that $L^{\prime} L^{-1} \in \tau_{0}$ and therefore $\left[L^{\prime} L^{-1}\right]_{\tau_{0}}=L^{\prime} L^{-1}$. Accordingly, an application of the operation $[\cdot]_{\tau_{0}}$ to (6) leads to $L^{\prime} L^{-1}=$ $\left[L A^{*} L^{-1}\right]_{\tau_{0}}$, and this is equivalent to (4).

To prove that the functions $F_{i k}$ in (5) are polynomials of degree not exceeding $n$, we observe that $L_{0}^{n}=0$ if $L_{0} \in \tau_{0}$. Thus, since $L_{0} \equiv I-L \in$ $\tau_{0}, L^{-1}=\left(I-L_{0}\right)^{-1}=I+L_{0}+\cdots+L_{0}^{n-1}$, and we have

$$
L A^{*} L^{-1}=\left(I-L_{0}\right) A^{*}\left(I+L_{0}+\cdots+L_{0}^{n-1}\right) .
$$

This shows that the elements of $L A^{*} L^{-1}$-and therefore also the elements of $\left[L A^{*} L^{-1}\right]_{\tau_{0}}$ are polynomials of degree $\leqslant n$ in the elements of $L_{0}$. By (4), the functions $F_{i k}$ in (5) are thus polynomials of degree $\leqslant n+1$ in the elements of $L_{0}$. The fact that the degree $n+1$ is excluded is a consequence of the identity

$$
\left[L_{0} A^{*} L_{0}^{n-1}\right]_{\tau_{0}} L=L_{0} A^{*} L_{0}^{n-1},
$$

which may be established in the following manner. Since $L_{0} \in \tau_{0}$, all elements of the matrix $L_{0}^{n-1}$, except the one in the lower left corner, are zero. As a result, the only nonzero elements of $A^{*} L_{0}^{n-1}$ appear in the first column, and we thus have $A^{*} L_{0}^{n-1} \in \tau$. This implies that $L_{0} A^{*} L_{0}^{n-1} \in \tau_{0}$, i.e., $\left[L_{0} A^{*} L_{0}^{n-1}\right]_{\tau_{0}}=L_{0} A^{*} L_{0}^{n-1}$. Hence,

as asserted.

$$
\left[L_{0} A^{*} L_{0}^{n-1}\right]_{\tau_{0}} L=L_{0} A^{*} L_{0}^{n-1}\left(I-L_{0}\right)=L_{0} A^{*} L_{0}^{n-1},
$$

We illustrate the use of Theorem I by two examples. The first is the case $n=2$, in which the matrix $L=\left(L_{i k}\right)_{i, k=1,2}$ has the elements $L_{11}=L_{22}=1$, $L_{12}=0, L_{21}=\rho$, where $\rho$ is a differentiable function of $t$. If $a_{i k}$ are the elements of the coefficient matrix $A$, a computation shows that the element appearing in the lower left corner of $\left[L A^{*} L^{-1}\right]_{\tau_{0}}$ is $\left(a_{11}-a_{22}\right) \rho+a_{12}-$ $a_{21} \rho^{2}$. Hence, (4) leads to the Riccati equation

$$
\rho^{\prime}=a_{12}+\left(a_{11}-a_{22}\right) \rho .-a_{21} \rho^{2} .
$$

By Theorem I, the interval $\left(t_{1}, t_{2}\right)$ will be free of points conjugate to $t_{1}$ with respect to the equation $x^{\prime}=A x$ if and only if the solution of (7) determined by $\rho\left(t_{1}\right)=0$ remains continuous in $\left(t_{1}, t_{2}\right)$. We also note that this example confirms the assertion, made above, that (4) reduces to the classical Riccati equation in the case $n=2$.

Our next example concerns the case $n=3$. To keep the formalism simple, we confine ourselves to a $3 \times 3$ system $x^{\prime}=A x$ which corresponds to 
the third-order linear differential equation

$$
u^{\prime \prime \prime}+p(t) u=0
$$

where $p$ is continuous on the interval considered. If we identify $x$, in the customary manner, with the vector $\left(u, u^{\prime}, u^{\prime \prime}\right)$, the nonzero elements $a_{i k}$, of the matrix $A$ are $a_{12}=1, a_{23}=1, a_{31}=-p$. If we denote the nontrivial elements of $L$ (i.e., the elements below the main diagonal) by $L_{21}=\rho$, $L_{31}=\sigma, L_{32}=\tau$, a computation shows that the nonzero elements of the matrix $B=\left[L A^{*} L^{-1}\right]_{\tau_{0}}$ are $B_{21}=1-p \rho(\rho \tau-\sigma), B_{31}=\tau-\rho-$ $p \sigma(\rho \tau-\sigma), B_{32}=1+p \sigma \tau$. Accordingly, (4) is found to be equivalent to the system of equations

$$
\rho^{\prime}=1-p \rho(\rho \tau-\sigma), \quad \sigma^{\prime}=\tau+p \sigma^{2}, \quad \tau^{\prime}=1+p \sigma \tau .
$$

These equations can be cast into a more symmetric form by introducing a redundant fourth function. If we set $\varphi=\rho \tau-\sigma$, it follows from (9) that $\varphi^{\prime}+p \varphi^{2}=\rho$. Accordingly, (9) is found to be equivalent to the system of four equations

$$
\begin{array}{ll}
\sigma^{\prime}=\tau+p \sigma^{2}, & \tau^{\prime}=1+p \sigma \tau, \\
\varphi^{\prime}=\rho-p \varphi^{2}, & \rho^{\prime}=1-p \rho \varphi
\end{array}
$$

and the relation $\varphi=\rho \tau-\sigma$.

By Theorem $I$, the nonexistence of a point $t_{3} \in\left(t_{1}, t_{2}\right)$ such that equation (8) has a solution $u$ with $u\left(t_{1}\right)=u^{\prime}\left(t_{1}\right)=u^{\prime \prime}\left(t_{2}\right)=0$ or $u\left(t_{1}\right)=u^{\prime}\left(t_{2}\right)=$ $u^{\prime \prime}\left(t_{2}\right)=0$ is equivalent to the existence of solutions $\sigma, \tau, \rho, \varphi$ of (10) which satisfy $\sigma\left(t_{1}\right)=\tau\left(t_{1}\right)=\rho\left(t_{1}\right)=\varphi\left(t_{1}\right)=0$ and are continuous on $\left[t_{1}, t_{2}\right)$.

We remark that in an interval $\left(t_{1}, t_{2}\right)$ in which they are continuous, these functions are necessarily positive. In the case of $\tau$ and $\rho$ this follows the fact that $\tau^{\prime}=\rho^{\prime}=1$ at $t_{1}$, which implies that $\tau>0$ and $\rho>0$ in a right neighborhood of $t_{1}$. At a first zero of $\tau$ (or $\rho$ ) in $\left(t_{1}, t_{2}\right)$ we would have $\tau^{\prime} \leqslant 0$ (or $\rho^{\prime} \leqslant 0$ ) which conflicts with the fact that, by $(10), \tau^{\prime}=1$ (or $\rho^{\prime}=1$ ) at such a point. In the case of $\sigma$ and $\varphi$ we have $\sigma^{\prime}\left(t_{1}\right)=\varphi^{\prime}\left(t_{1}\right)=$ 0 , and (10) shows that $\left(t-t_{0}\right)^{-1} \tau(t)$ and $\left(t-t_{0}\right)^{-1} \varphi(t)$ tend to 1 if $t \rightarrow t_{0}$ from the right, so that $\sigma$ and $\varphi$ are again positive in a right neighborhood of $t_{1}$. At a first zero of these functions in $\left(t_{1}, t_{2}\right)$ we have, by (10), $\sigma^{\prime}=\tau$ and $\varphi^{\prime}=\rho$, respectively. Since $\rho>0$ and $\tau>0$ in $\left(t_{1}, t_{2}\right)$, we obtain the same contradiction as before.

In the case in which the coefficient function $p$ is of constant sign, the positivity of the functions $\tau, \sigma, \rho, \varphi$ leads to a considerable simplification. If $p \geqslant 0$, it follows from the equations (10b) that $\varphi^{\prime} \leqslant \rho, \rho^{\prime} \leqslant 1$, and thus $0 \leqslant \rho \leqslant t, 0 \leqslant \varphi \leqslant 1 / 2 t^{2}$, as long as $\rho$ and $\varphi$ are defined. We may therefore conclude from the existence theorem that the differential equations (10b) (with 
the initial conditions $\rho\left(t_{1}\right)=\varphi\left(t_{1}\right)=0$ ) have a unique and continuous pair of solutions $\rho, \varphi$ as long as $p$ remains continuous. Hence, these equations may be disregarded, and our necessary and sufficient condition for the absence in $\left(t_{1}, t_{2}\right)$ of a point conjugate to $t_{1}$ reduces to the existence in $\left[t_{1}, t_{2}\right)$ of a continuous solution of the system (10a) with the initial conditions $\sigma\left(t_{1}\right)=$ $\tau\left(t_{1}\right)=0$. In the case $p \leqslant 0$ we have a similar result, with (10a) replaced by $(10 b)$.

We note that the two equations (10a) are equivalent to equation (8). Indeed if we set $\exp \left[-\int_{0}^{t} p \sigma d s\right]=R$, the equations (10a) take the form $(R \sigma)^{\prime}$ $=R \tau,(R \tau)^{\prime}=R$. With $u=R \sigma$, we have therefore $u^{\prime}=R \tau, u^{\prime \prime}=R$. Since $R^{\prime}=-p \sigma R=-p u$, we thus find that $u$ satisfies equation (8). Similarly, the equations (10b) are equivalent to the equation $v^{\prime \prime \prime}-p v=0$.

The classical Riccati equation is a convenient point of departure for the derivation of oscillation criteria for the associated second-order linear equation. The "Riccati system" (10a) can be made to play a similar role in the oscillation theory of equation (8). To illustrate the possibilities, we consider the case $p \geqslant$ 0 and the interval $[0, \infty)$. It is known [2] that in this case a conjugate point $t_{0}$ of 0 (in the sense defined above) is necessarily associated with a solution of (8) for which $u(0)=u^{\prime}(0)=u^{\prime \prime}\left(t_{0}\right)=0$; moreover, the absence on $[0, \infty)$ of a point conjugate to 0 is equivalent to the disconjugacy of the equation in the sense of Wintner [2], i.e., to the fact that no nontrivial solution of (8) can have more than two zeros on $[0, \infty)$.

If we set $T=\sigma / \tau$, it follows from (10a) that $T^{\prime}=1-T \tau^{-1}$. Since, by the second equation (10a), $\tau \geqslant t$ we have $T^{\prime} \geqslant 1-T t^{-1}$, i.e., $(t T)^{\prime} \geqslant t$, and thus $T \geqslant 1 / 2 t$. On the other hand, $T^{\prime} \leqslant 1$, and therefore (because of $T(0)=$ $0), T \leqslant t$. Applying these inequalities to the second equation (10a), we obtain $1+1 / 2 p t \tau^{2} \leqslant \tau^{\prime} \leqslant 1+p t \tau^{2}$. If we set $\tau=w\left(w^{\prime}\right)^{-1}$, where $w(0)=0, w^{\prime}(0)>0$, these inequalities take the form $w^{\prime \prime}+1 / 2 p t w \leqslant 0 \leqslant w^{\prime \prime}+p t w$, and the continuity of $\tau$ (and, because of $0 \leqslant \sigma \leqslant \tau$, also the continuity of $\sigma$ ) is equivalent to $w \neq 0$. Elementary comparison arguments (cf., e.g., [15]) show that the existence of such a function $w$ implies the existence of a solution $U$ of $U^{\prime \prime}+1 / 2 p t U=0$ with $U(0)$ $=0, U^{\prime} \neq 0$, and is implied by the existence of a solution $V$ of $V^{\prime \prime}+p t V=0$ with $V(0)=0, V^{\prime} \neq 0$ (both on the interval $[0, \infty)$ ). Since the existence of these solutions is equivalent to the disconjugacy of these equations on $[0, \infty)[15]$, this leads to the following result.

In order that equation (8) (with $p \geqslant 0$ ) be disconjugate on $[0, \infty)$, it is necessary that $U^{\prime \prime}+1 / 2 p t U=0$ be disconjugate on $[0, \infty)$ and sufficient that $U^{\prime \prime}+$ $p t U=0$ be disconjugate on this interval.

3. Let $T(t)$ be a differentiable matrix function on $\left[t_{1}, t_{2}\right]$ such that 
$T\left(t_{1}\right) \in \tau$ and $T\left(t_{2}\right) \in \tau^{*}$, and let $Y^{\prime}=A_{1} Y$ be the differential equation solved by $Y=T X$, where $X$ is the solution of (2) described in Lemma I. It is evident from Lemma I that $t_{2}$ will not be a conjugate point of $t_{1}$ with respect to the transformed equation if the same is true of equation (2), and vice versa. Since $A_{1}=\left(T^{\prime}+T A\right) T^{-1}$, Lemma I has the following consequence.

LEMMA III. In order that $t_{2}$ be not a conjugate point of $t_{1}$ with respect to equation (2), it is necessary and sufficient that the same be true for the equation

$$
Y^{\prime}=A_{1} Y, \quad A_{1}=\left(T^{\prime}+T A\right) T^{-1},
$$

where $T$ is any nonsingular matrix which is differentiable on $\left[t_{1}, t_{2}\right]$ and satisfies the conditions $T\left(t_{1}\right) \in \tau, T\left(t_{2}\right) \in \tau^{*}$.

A similar conclusion may be drawn from Lemma II. If $X$ is the solution described in Lemma II and $R$ is a matrix which is differentiable and $\in \tau$ on $\left[t_{1}, t_{2}\right]$ and reduces to the unit matrix at $t_{1}$, the matrix $Y=R^{*} X=R^{*} L^{*} S$ satisfies all the conditions imposed on $X$ in Lemma II, and we have the following result.

Lemma IV. In order that the interval $\left(t_{1}, t_{2}\right]$ should not contain a conjugate point of $t_{1}$ with respect to equation (2), it is necessary and sufficient that the same be true for the equation

$$
Y^{\prime}=A_{2} Y, \quad A_{2}=\left(R^{* \prime}+R^{*} A\right) R^{*-1},
$$

where $R$ is any matrix of $\tau$ which is differentiable on $\left[t_{1}, t_{2}\right]$ and such that $R\left(t_{1}\right)=I$.

These lemmas show that any condition on the coefficient matrix $A$ which guarantees the existence or nonexistence of conjugate points can be replaced by the same condition on the matrices $A_{1}$ or $A_{2}$, as the case may be. Since the latter matrices depend on arbitrary triangular matrices, this leads to conditions of considerable generality. We shall illustrate this remark in the case of the condition

$$
\int_{t_{1}}^{t_{2}}\|A\| d t<\frac{\pi}{2}
$$

which guarantees that the interval $\left(t_{1}, t_{2}\right]$ does not contain a point conjugate to $t_{1}$ with respect to equation (2) [6], [10]. Here, $\|\cdot\|$ denotes the matrix norm induced by the euclidean vector norm. (Conditions employing other norms can be found in [11], [13].) We also note that the constant $\pi / 2$ in (12) is the largest possible; in fact, the stated consequence does not follow if the sign of equality is admitted in (12) [10].

To make this paper self-contained, we give here a very simple derivation of condition (12). Introducing the variable $s=s(t)=\int_{t_{1}}^{t}\|A\| d t$ and writing 
$\dot{x}$ for $d x / d s$, we obtain from (1)

$$
\|A\|\|\dot{x}\|=\left\|x^{\prime}\right\|=\|A x\| \leqslant\|A\|\|x\|,
$$

and thus $\|\dot{x}\| \leqslant\|x\|$. With $x=\left(x_{1}, \cdots, x_{n}\right)$ it follows that

$$
\sum_{k=1}^{n}\left[\int_{0}^{\alpha} \dot{x}_{k}^{2} d s-\int_{0}^{\alpha} x_{k}^{2} d s\right] \leqslant 0, \quad \alpha=s\left(t_{3}\right), \quad t_{3} \in\left(t_{1}, t_{2}\right],
$$

and we may therefore conclude that there exists a component $x_{k}$ for which

$$
\int_{0}^{\alpha} \dot{x}_{k}^{2} d s \leqslant \int_{0}^{\alpha} x_{k}^{2} d s
$$

If $t_{3}$ is conjugate to $t_{1}$, there exists a solution $x$ such that each of its components vanishes at either $t_{1}$ or $t_{3}$, and we may therefore assume that the function $x_{k}=x_{k}(s)$ is such that either $x_{k}(0)=0$ or $x_{k}(\alpha)=0$. On the other hand, it is well known (cf., e.g., [3]) that a function with these properties is subject to the inequality $\int_{0}^{\alpha} x_{k}^{2} d s<\int_{0}^{\alpha} \dot{x}_{k}^{2} d s$, provided $\alpha<\pi / 2$. Since this contradicts (13), it follows that $t_{3}$ cannot be conjugate to $t_{1}$ if $\pi / 2>\alpha$ $=s\left(t_{3}\right)=\int_{t_{1}}^{t_{2}}\|A\| d t$. Hence, (12) guarantees that no point in $\left(t_{1}, t_{2}\right]$ is conjugate to $t_{1}$.

Combining condition (12) with Lemmas III and IV, we obtain the following two results.

THEOREM II. If there exists a differentiable nonsingular matrix $T$ on $\left[t_{1}, t_{2}\right]$ such that $T\left(t_{1}\right) \in \tau, T\left(t_{2}\right) \in \tau^{*}$ and $\int_{t_{1}}^{t_{2}}\left\|\left(T^{\prime}+T A\right) T^{-1}\right\| d t<\pi / 2$, then $t_{2}$ cannot be a conjugate point to $t_{1}$ with respect to equation (2).

THEOREM III. If there exists a differentiable matrix $R \in \tau$ on $\left[t_{1}, t_{2}\right]$ such that $R\left(t_{1}\right)=I$ and

$$
\int_{t_{1}}^{t_{2}}\left\|R^{-1}\left(R^{\prime}+A^{*} R\right)\right\| d t<\frac{\pi}{2},
$$

then the interval $\left(t_{1}, t_{2}\right]$ does not contain a point conjugate to $t_{1}$ with respect to equation (2).

Every choice of a matrix $T$ or $R$ with the requisite properties thus leads to an explicit criterion. In the case $n=2$, the situation is particularly favorable. As the following statement shows, in this case all possible disconjugacy criteria can be obtained in this way. We confine ourselves here to the situation covered by Theorem II; the corresponding assertion related to Theorem I follows as a corollary.

Theorem IV. Let $A=\left(a_{i k}\right)$ be a $2 \times 2$ continuous matrix such that $a_{21} \neq 0$ on $\left[t_{1}, t_{2}\right]$. In order that the interval $\left(t_{1}, t_{2}\right]$ contain no point conjugate to $t_{1}$ with respect to equation (2) it is necessary and sufficient that 
there exist a differentiable $2 \times 2$ matrix $R \in \tau$ which satisfies the inequality (14) and the initial condition $R\left(t_{1}\right)=I$.

All we have to show is that if $a_{21} \neq 0$ and the interval $\left(t_{1}, t_{2}\right]$ contains no point conjugate to $t_{1}$, there exists a matrix $R \in \tau$ such that $R\left(t_{1}\right)=$ $I$ and (14) is true. To do so, we denote by $X$ the solution of (2) determined by the initial condition $X\left(t_{1}\right)=I$ and apply the Schmidt decomposition (cf., e.g., $\left[16\right.$, p. 96]) to the matrix $X^{*-1}$. We obtain $X^{*-1}=R Q$, where $R \in$ $\tau$ (since $X^{*-1}$ is nonsingular) and $Q$ is an orthogonal matrix. If the diagonal elements of $R$ are taken positive, both $R$ and $Q$ are uniquely determined (and are differentiable since $X$ is differentiable). From the fact that $R Q=I$ at $t_{1}$ it follows that $R=I$ at $t_{1}$. Indeed, we have $I=(R Q)(R Q)^{*}=R R^{*}$, i.e., $R=R^{*-1}$. Since $R \in \tau, R^{*-1} \in \tau^{*}$, this implies that $R$ is a diagonal matrix. But $I=R R^{*}=R^{2}$, and the diagonal elements of $R$ are positive. Hence, $X^{*-1}=R Q$, with $R\left(t_{1}\right)=I$. Solving for $Q$ (and using the fact that $\left.Q^{*}=Q^{-1}\right)$, we have $Q=R^{*} X$ and therefore

$$
Q^{\prime} Q^{-1}=\left(R^{* \prime}+R^{*} A\right) R^{*-1}=A_{2},
$$

where $A_{2}$ is the matrix (11). Hence, by Lemma IV, the absence in $\left(t_{1}, t_{2}\right]$ of a point conjugate to $t_{1}$ with respect to equation (2) is equivalent to the same property with respect to the equation

Since $Q$ is orthogonal, we have

$$
Q^{\prime}=A_{2} Q
$$

$$
A_{2}+A_{2}^{*}=Q^{\prime} Q^{-1}+\left(Q^{\prime} Q^{-1}\right)^{*}=Q^{\prime} Q^{*}+Q Q^{* \prime}=\left(Q Q^{*}\right)^{\prime}=0 .
$$

Thus, the coefficient matrix $A_{2}$ in (11) is skew-symmetric.

We remark that this property of $A_{2}$ leads to a differential equation for the triangular matrix $R$. Since $A_{2}$ may be written in the form $S^{*}-S$, where $S \in \tau_{0}$, we have, by (11), $R^{-1}\left(R^{\prime}+A^{*} R\right)=S-S^{*}$. Applying successively the operations []$_{\tau}$ and []$_{\tau_{0}^{*}}$ and noting that $\left[R^{-1} R^{\prime}\right]_{\tau}=R^{-1} R^{\prime}$, we obtain $R^{-1} R^{\prime}+\left[R^{-1} A^{*} R\right]_{\tau}=S$ and $\left[R^{-1} A^{*} R\right]_{\tau_{0}^{*}}=-S^{*}$. Eliminating $S$, we find that $R$ is the solution of the differential equation

$$
R^{\prime}=-R\left\{\left[R^{-1} A^{*} R\right]_{\tau}+\left(\left[R^{-1} A^{*} R\right]_{\tau_{0}^{*}}\right)^{*}\right\}
$$

determined by the initial condition $R\left(t_{1}\right)=I$. It may be noted that although this equation is not linear, the solution $R$ is-because of its definition by means of the Schmidt decomposition-necessarily continuous throughout the interval of continuity of $A$.

If $n=2$, the skew-symmetric matrix $A_{2}=\left(b_{i k}\right)$ has the elements $b_{11}=$ $b_{22}=0, b_{12}=\varphi, b_{21}=-\varphi$, where $\varphi$ is a continuous scalar function on $\left[t_{1}, t_{2}\right]$. The solution $x=\left(x_{1}, x_{2}\right)$ of the vector-matrix equation associated with (15) for which $x\left(t_{1}\right)=(0,1)$ is 


$$
x=\left[\sin \int_{t_{1}}^{t} \varphi d s, \cos \int_{t_{1}}^{t} \varphi d s\right],
$$

and this shows that the conjugate point of $t_{1}$ is the point $t_{3}\left(t_{3}>t_{1}\right)$ for which $\int_{t_{1}}^{t_{3}} \varphi d s=\pi / 2$. Since $\left\|A_{2}\right\|=|\varphi|$, the absence on $\left(t_{1}, t_{2}\right]$ of a point conjugate to $t_{1}$ will therefore imply $\int_{t_{1}}^{t_{2}}\left\|A_{2}\right\| d s<\pi / 2$ (i.e., because of (11), the inequality (14)), provided $\varphi$ is of constant sign. Accordingly, Theorem IV will be proved if we can show that $\varphi \neq 0$ on $\left[t_{1}, t_{2}\right]$ if the element $a_{21}$ of $A$ does not vanish on this interval. Using (11) and the special form of $A_{2}$ in our case, we have

$$
\left(\begin{array}{ll}
R_{11}^{\prime} & 0 \\
R_{21}^{\prime} & R_{22}^{\prime}
\end{array}\right)+\left(\begin{array}{ll}
a_{11} & a_{21} \\
a_{12} & a_{22}
\end{array}\right)\left(\begin{array}{ll}
R_{11} & 0 \\
R_{21} & R_{22}
\end{array}\right)=\left(\begin{array}{ll}
R_{11} & 0 \\
R_{21} & R_{22}
\end{array}\right)\left(\begin{array}{ll}
0 & -\varphi \\
\varphi & 0
\end{array}\right) .
$$

Comparing the elements in the upper right corner, we obtain $a_{21} R_{22}=-\varphi R_{11}$. Since $R \in \tau$, we have $R_{11}, R_{22} \neq 0$, and $\varphi \neq 0$ is thus a consequence of the assumption $a_{21} \neq 0$. This completes the proof of Theorem IV.

To illustrate the nature of the formalism involved in the nonlinear equation (16), we compute $R$ in the case of a $2 \times 2$ system corresponding to the second-order equation $y^{\prime \prime}+p y=0, p>0$. With the coefficient matrix $\left(a_{i k}\right)$, where $a_{11}=a_{22}=0, a_{12}=1, a_{21}=-p$, and the abbreviations $R_{11}=\rho$, $R_{21}=\sigma, R_{22}=\tau,(16)$ is found to lead to the system of differential equations

$$
\rho^{\prime}=p \sigma, \quad \sigma^{\prime}=\frac{p \tau^{2}}{\rho}-\rho, \quad \tau^{\prime}=-\frac{p \sigma \tau}{\rho} .
$$

The first and third equations lead to $(\rho \tau)^{\prime}=0$ and therefore, because of $R\left(t_{1}\right)=I$, to $\rho \tau=1$. Using this, and eliminating $\tau$ and $\sigma$, we find that $\rho$ must be a solution of $\left(\rho^{\prime} / p\right)^{\prime}+\rho=p / \rho^{3}$. Now it is easily confirmed that the general solution of this equation is of the form $\rho=\left(u^{2}+v^{2}\right)^{1 / 2}$, where $u$ and $v$ are two linearly independent solutions of the linear differential equation

$$
\left(w^{\prime} / p\right)^{\prime}+w=0
$$

which are normalized by the condition $v u^{\prime}-u v^{\prime}=p$ (the fact that $v u^{\prime}-u v^{\prime}$ $=\alpha p, \alpha$ constant, follows from Abel's identity). It may be noted that, because $\rho=\left(u^{2}+v^{2}\right)^{1 / 2}>0$, the solutions $\rho, \sigma, \tau$ of (17) are continuous if $p$ is continuous, as expected. (16) also shows that $\varphi=p \rho^{-2}$. Since $v u^{\prime}-u v^{\prime}=$ $p$, we therefore have

and thus

$$
\varphi=\frac{p}{\rho^{2}}=\frac{p}{u^{2}+v^{2}}=\frac{(u / v)^{\prime}}{1+(u / v)^{2}}
$$

$$
\int_{t_{1}}^{t_{2}} \varphi d s=\left[\arctan \frac{u}{v}\right]_{t_{1}}^{t_{2}}
$$


Since $u, v$ are solutions of (18), the functions $U=u^{\prime} p^{-1}, V=v^{\prime} p^{-1}$ are solutions of the original equation $y^{\prime \prime}+p y=0$, which are normalized by the condition $V U^{\prime}-U V^{\prime}=1$, and (19) may be replaced by

$$
\int_{t_{1}}^{t_{2}} \varphi d s=\left[\arctan \frac{U^{\prime}}{V^{\prime}}\right]_{t_{1}}^{t_{2}}
$$

If we define $U, V$ by the initial conditions $U\left(t_{1}\right)=0, U^{\prime}\left(t_{1}\right)=1, V\left(t_{1}\right)=1$, $V^{\prime}\left(t_{1}\right)=0$, we have $V^{\prime}<0$ in a right neighborhood of $t_{1}$, and therefore $U^{\prime}\left(V^{\prime-1}\right) \rightarrow-\infty$ if $t \rightarrow t_{1}$ from the right. Since the conjugate point $t_{2}$ of $t_{1}$ is the first zero of $U^{\prime}$, the integral (20) has the value $\pi / 2$; because of $\varphi>0$, its value is $<\pi / 2$ if its upper limit is a point in $\left(t_{1}, t_{2}\right)$.

4. In the case in which $A$ is the companion matrix associated with an $n$th order differential equation

$$
y^{(n)}+p_{n-1} y^{(n-1)}+\cdots+p y=0,
$$

a conjugate point $t_{2}$ of $t_{1}$ with respect to the equation $x^{\prime}=A x$ is characterized by the existence of a nontrivial solution $y$ of $(21)$ for which $y\left(t_{1}\right)=y^{\prime}\left(t_{1}\right)=\cdots=$ $y^{(k-1)}\left(t_{1}\right)=y^{(k)}\left(t_{2}\right)=\cdots=y^{(n-1)}\left(t_{2}\right)=0$, where $k \in[1, n-1]$. In the terminology used in the theory of equation (21), such a point is called a focal point, and the term "conjugate point" is reserved for a point $t_{2}$ such that (21) has a nontrival solution $y$ with $y\left(t_{1}\right)=y^{\prime}\left(t_{1}\right)=\cdots=y^{(k-1)}\left(t_{1}\right)=y\left(t_{2}\right)=y^{\prime}\left(t_{2}\right)=\cdots=y^{(n-k-1)}\left(t_{2}\right)$ $=0$ [4] , [5] , [7] , [8] , [9] , [14]. In the corresponding vector-matrix equation $x^{\prime}=A x$, the latter type of point is characterized by the existence of a nontrivial solution $x=\left(x_{1}, \cdots, x_{n}\right)$ such that $x_{1}\left(t_{1}\right)=x_{2}\left(t_{1}\right)=\cdots=x_{k}\left(t_{1}\right)=0, x_{1}\left(t_{2}\right)=$ $x_{2}\left(t_{2}\right)=\cdots=x_{n-k}\left(t_{2}\right)=0, k \in[1, n-1]$. To avoid confusion we shall call a point of this type a "conjugate point of the second kind" with respect to equation (1). While this concept is of particular interest in the case in which the coefficient matrix $A$ is the companion matrix of an equation (21), it can be applied to the general equation (1).

The following result shows that the absence of a conjugate point of the second kind is equivalent to the possibility of decomposing a certain fundamental solution matrix $X$ of (2) into a product to two triangular matrices. The situation is similar to that described in Lemma II, but there are two significant differences: The order of the two triangular matrices is reversed, and the solution $X$ now has a different initial value.

LEMMA V. Let $E=\left(E_{i k}\right)$ be the $n \times n$ matrix whose only nonvanishing elements are $E_{k, n+1-k}=1, k=1, \cdots, n$. In order that the interval $\left(t_{1}, t_{3}\right)$ be free of conjugate points of the second kind $t_{2}\left(t_{1}\right)$ with respect to equation (1), it is necessary and sufficient that the solution $X$ of (2) with the initial value $X\left(t_{1}\right)$ 


\section{$=E$ have a representation}

$$
X=L S^{*}, \quad L, S \in \tau,
$$

where the matrices $L, S$ are differentiable on $\left(t_{1}, t_{2}\right)$.

The representation (22) can be made unique by requiring, for example, that the elements in the main diagonal of $L$ be all equal to 1 .

The exclusion of the point $t_{1}$ from the interval of differentiability-and, indeed, continuity-of $L$ and $S$ is unavoidable. If these matrices were continuous on $\left[t_{1}, t_{2}\right)$, it would follow from $L\left(t_{1}\right) S^{*}\left(t_{2}\right)=E$ that both $L\left(t_{1}\right)$ and $S\left(t_{1}\right)$ are nonsingular (and thus $\in \tau$ ), and therefore $L\left(t_{1}\right)=E S^{*-1}\left(t_{1}\right)$. Since the elements of $E S^{*-1}$ above the secondary diagonal are all zero, this would imply that the elements of $L\left(t_{1}\right)$ along the upper half of the main diagonal are all zero, and this is incompatible with $L\left(t_{1}\right) \in \tau$. The matrix functions $L(t)$ and $S(t)$ may thus be expected to exhibit singular behavior (singular in the analytic, not the algebraic, sense) as $t \rightarrow t_{1}$ from the right. The nature of this singular behavior will be discussed later.

We now turn to the proof of Lemma V. Suppose the point $t_{2}=t_{2}\left(t_{1}\right)$ is a conjugate point of the second kind, i.e.-in the terminology used in the proof of Lemma II-suppose there exists a nontrivial solution $x$ of (1) such that, for some $k \in[1, n-1], x\left(t_{1}\right) \in N_{k}$ and $x\left(t_{2}\right) \in N_{n-k}$. Let $X$ be the solution of (2) for which $X\left(t_{1}\right)=E$, and suppose that $X$ has the representation (22). Since $X$ is a fundamental solution, we have $x=X \alpha$, where $\alpha$ is a constant vector, and therefore

$$
E^{-1} x\left(t_{1}\right)=X^{-1}\left(t_{1}\right) x\left(t_{1}\right)=X^{-1}\left(t_{2}\right) x\left(t_{2}\right)=S^{*-1}\left(t_{2}\right) L^{-1}\left(t_{2}\right) x\left(t_{2}\right),
$$

or (because of $E^{-1}=E$ )

$$
S^{*}\left(t_{2}\right) \operatorname{Ex}\left(t_{1}\right)=L^{-1}\left(t_{2}\right) x\left(t_{2}\right) .
$$

Since $x\left(t_{2}\right) \in N_{n-k}$ and $L^{-1}\left(t_{2}\right) \in \tau$, the right-hand side of (23) is also a vector of class $N_{n-k}$. On the other hand, multiplication of a vector by the matrix $E$ reverses the order of its components, and it follows from $x\left(t_{1}\right) \in N_{k}$ that $\operatorname{Ex}\left(t_{1}\right) \in N_{k}^{\prime}$. Because of $S^{*}\left(t_{2}\right) \in \tau^{*}$, the left-hand side of (23) is thus found to be a vector of the class $N_{k}^{\prime}$. As remarked earlier, the only vector $\beta$ for which both $\beta \in N_{k}^{\prime}$ and $\beta \in N_{n-k}$ is the zero vector. Thus, both sides of (23) are zero. Since both $S^{*}\left(t_{2}\right)$ and $E$ are nonsingular, it follows that $x\left(t_{1}\right)=0$, i.e., $x$ is the trivial solution of (1), contrary to our assumption. This shows that conjugate points of the second kind cannot occur if the solution $X$ has the representation(22).

To prove the converse, suppose that $t_{2}$ is not a conjugate point of the second kind (of $t_{1}$ ), and let $x$ be a nontrivial solution of (1) for which $x\left(t_{1}\right) \in N_{k}$, where $k$ may be any integer in $[1, n-1]$. If $X$ is the solution of (1) for which $X\left(t_{1}\right)=$ $E$, we then have $X^{-1}\left(t_{1}\right) x\left(t_{1}\right)=E x\left(t_{1}\right) \in N_{k}^{\prime}$. Since $X^{-1}(t) x(t)=\alpha=$ const, it 
follows that $x\left(t_{2}\right)=X\left(t_{2}\right) \alpha$, where $\alpha \in N_{k}^{\prime}$ and the components $\alpha_{1}, \alpha_{2}, \cdots$, $\alpha_{n-k-1}$ of $\alpha$ may be given arbitrary values by imposing suitable initial conditions on the components $x_{k+1}, x_{k+2}, \cdots, x_{n}$ of $x$. By assumption, $x\left(t_{2}\right) \notin$ $N_{n-k}$ for all nontrivial choices of $\alpha_{1}, \cdots, \alpha_{n-k-1}$, and this implies that the $(n-k) \times(n-k)$ submatrix of $X\left(t_{2}\right)$ in the upper left corner is nonsingular. Since $k$ may be any integer in $[1, n-1]$, all square submatrices of $X\left(t_{2}\right)$ which contain the element in the upper left corner of $X\left(t_{2}\right)$ are thus found to be nonsingular, and it follows by the result quoted above that $X\left(t_{2}\right)=L S^{*}$, where $L, S \in \tau$. If this decomposition is made unique by setting the elements in the main diagonal of $L$ equal to 1 , the differentiability of $L$ and $S$ becomes a consequence of the differentiability of $X$ (cf. the proof of Lemma II). This completes the proof of Lemma V.

We now apply to (22) a procedure similar to that which led from the decomposition (3) to the differential equation (4). Since, by (22) and (2), $L S^{*}+L S^{* \prime}=$ $A L S^{*}$, we have

$$
L^{-1} L^{\prime}+S^{* \prime} S^{*-1}=L^{-1} A L .
$$

Applying to this the operation []$_{\tau_{0}}$, we obtain $\left[L^{-1} L^{\prime}\right]_{\tau_{0}}=\left[L^{-1} A L\right]_{\tau_{0}}$. If $L$ is normalized so that all the elements in its main diagonal are 1 , we clearly have $L^{\prime} \in \tau_{0}$ and therefore $L^{-1} L^{\prime} \in \tau_{0}$. Thus, $\left[L^{-1} L^{\prime}\right]_{\tau_{0}}=L^{-1} L^{\prime}$, and the differential equation for $L$ simplifies to

$$
L^{\prime}=L\left[L^{-1} A L\right]_{\tau_{0}}
$$

A comparison with (4) shows that, though of similar build, this equation is essentially different from (4). The equations also differ in the initial conditions to be satisfied by the solution $L$. In the case of equation (4), all nontrivial elements of $L$ had the initial value 0 . In the case of (25), the situation is more complicated since, as pointed out above, some of the elements of $L$ must become singular as $t \rightarrow t_{1}$ from the right.

By way of illustration, we consider the case of a $2 \times 2$ matrix $A=\left(a_{i k}\right)$. Clearly, the only nontrivial element of $L$ is now $L_{21}$, and a short computation shows that (25) is in this case equivalent to the Riccati equation

$$
R^{\prime}=a_{21}+\left(a_{22}-a_{11}\right) R-a_{12} R^{2}
$$

for $R=L_{21}$. (It may be noted that (26) transforms into (7) under the substitution $R^{-1}=\rho$.) To find the initial conditions to be satisfied by $R$ we note that, for small values of $\epsilon=t-t_{1}$,

$$
L(t) S^{*}(t)=X(t)=E+\int_{t_{1}}^{t} A(s) E d s+O\left(\epsilon^{2}\right) .
$$

Denoting the elements of $S$ by $S_{i k}$, we obtain 


$$
S_{11}=\int_{t_{1}}^{t} a_{12} d s+O\left(\epsilon^{2}\right), \quad R S_{11}=1+\int_{t_{1}}^{t} a_{22} d s+L\left(\epsilon^{2}\right)
$$

by equating the elements in the first column. Hence, $\lim _{t \rightarrow t_{1}}\left(t-t_{1}\right) R(t)=\left[a_{12}\left(t_{1}\right)\right]^{-1}$ (the fact that $a_{12}\left(t_{1}\right) \neq 0$ is a consequence of $S \in \tau$ ). Our final result is therefore that the interval $\left(t_{1}, t_{2}\right)$ is free of conjugate points of the second kind if and only if the Riccati equation (26) has a solution which is continuous in $\left(t_{1}, t_{2}\right)$ and is such that $\lim _{t \rightarrow t_{1}}\left(t-t_{1}\right) R(t)$ exists and is $\neq 0$. That this limit must have the value $\left[a_{12}\left(t_{1}\right)\right]^{-1}$ can be seen by setting $R^{-1}=\rho$ and using equation (7).

In the further discussion of equation (25) we confine ourselves to the case in which $A$ is the companion matrix of the $n$th order differential equation

$$
y^{(n)}-p_{n-2} y^{(n-2)}-p_{n-3} y^{(n-3)}-\cdots-p_{1} y^{\prime}-p_{0} y=0 .
$$

In this case (which is the one of major interest), the particular form of $A$ leads to a considerable simplification of the procedure for obtaining the correct initial conditions for the solution matrix $L$ of (25). Also, for matrices $A$ of this type equation (25) can be brought into a very much simpler form.

The companion matrix of equation (27) is $A=A_{0}+E_{1}$, where the elements in the bottom row of $A_{0}$ are $p_{0}, p_{1}, \cdots, p_{n-2}, 0$, and all other elements of $A_{0}$ are zero; $E_{1}$ is the matrix $\left(b_{i k}\right)$ whose only nonzero elements are $b_{i, i+1}=1$, $i=1,2, \cdots, n-1$. We shall show that for such a matrix $A$ equation(25) reduces to

$$
L^{\prime}=A L-L E_{1}+L\left(L_{0} E_{1}-E_{1} L_{0}\right)
$$

where $L_{0}=\left(c_{i k}\right)$ is the matrix whose only nonzero elements are $c_{i, i+1}=L_{i, i+1}$, $i=1, \cdots, n-1$ (and $L_{i k}$ are the elements of $L$ ). Written in terms of the $L_{i k}$, (28) is equivalent to the system

$$
L_{i k}^{\prime}=L_{i+1, k}-L_{i, k+1}+L_{i k}\left(L_{k, k-1}-L_{k+1, k}\right),
$$

$$
\begin{array}{r}
i, k=1, \cdots, n-1,\left(L_{i 0}=0\right), \\
L_{n k}^{\prime}=p_{k-1}+p_{k} L_{k+1, k}+\cdots+p_{n-2} L_{n-1, k}+L_{n k}\left(L_{k, k-1}-L_{k+1, k}\right), \\
\left(L_{n+1, n}=0\right) .
\end{array}
$$

To derive (28), we observe that $A_{0} \in \tau_{0}$ and therefore (because of $L \in \tau$ ) $L^{-1} A_{0} L \in \tau_{0}$. Hence, $\left[L^{-1} A L\right]_{\tau_{0}}=L^{-1} A L-\left[L^{-1} A L\right]_{\tau^{*}}=L^{-1} A L-$ $\left[L^{-1}\left(A_{0}+E_{1}\right) L\right]_{\tau^{*}}=L^{-1} A L-\left[L^{-1} E_{1} L\right]_{\tau^{*}}$, and (25) is found to be equivalent to

$$
L^{\prime}=A L-L\left[L^{-1} E_{1} L\right]_{\tau^{*}}
$$

We introduce here, for the moment, the notation $\tau_{1}$ for the class of matrices $\left(\eta_{i k}\right)$ for which $\eta_{i k}=0$ if $k>i-1$. Clearly, $L-I-L_{0} \in \tau_{1}$ and, as a result, $E_{1}\left(L-I-L_{0}\right) \in \tau_{0}$ and $L^{-1} E_{1}\left(L-I-L_{0}\right) \in \tau_{0}$. Hence,

$$
\left[L^{-1} E_{1} L\right]_{\tau^{*}}=\left[L^{-1} E_{1}\left(I+L_{0}\right)+L^{-1} E_{1}\left(L-I-L_{0}\right)\right]_{\tau^{*}}=\left[L^{-1}\left(I+L_{0}\right)\right]_{\tau^{*}} \text {. }
$$


From $L-I \in \tau_{0},(L-I)^{2} \in \tau_{1}$ and $L^{-1}=I-(L-I)+\cdots+(-1)^{n-1}(L-I)^{n-1}$ it follows that $L^{-1}-I+L_{0} \in \tau_{1}$ and thus, as before, $\left(L^{-1}-I+L_{0}\right) E_{1}\left(L-I-L_{0}\right)$ $\in \tau_{0}$. This implies that $\left[L^{-1} E_{1}\left(I+L_{0}\right)\right]_{\tau^{*}}=\left[\left(I-L_{0}\right) E_{1}\left(I+L_{0}\right)\right]_{\tau^{*}}$. Since, as just shown, $\left[L^{-1} E_{1} L\right]_{\tau^{*}}=\left[L^{-1} E_{1}\left(I+L_{0}\right)\right]_{\tau^{*}}$, we find that (29) reduces to

$$
L^{\prime}=A L-L\left[\left(I-L_{0}\right) E_{1}\left(I+L_{0}\right)\right]_{\tau^{*}} .
$$

Since $L_{0} E_{1} L_{0} \in \tau_{0}, E_{1} \in \tau^{*}$, and both $L_{0} E_{1}$ and $E_{1} L_{0}$ are diagonal matrices, we have $\left[\left(I-L_{0}\right) E_{1}\left(I+L_{0}\right)\right]_{\tau^{*}}=E_{1}+E_{1} L_{0}-L_{0} E_{1}$, and this establishes (28). We also note that (24) and the identity $\left[L^{-1} A L\right]_{\tau^{*}}=\left[L^{-1} E_{1} L\right]_{\tau^{*}}=E_{1}+E_{1} L_{0}$ $-L_{0} E_{1}$ lead to the differential equation

$$
S^{* \prime}=\left(E_{1}+E_{1} L_{0}-L_{0} E_{1}\right) S^{*}
$$

for the matrix $S$.

We now consider the behavior of the elements of $L$ as $t \rightarrow t_{1}$. Since $A$ is the companion matrix of the equation (27), the matrix $X$ described in Lemma $\mathrm{V}$ is the "Wronskian matrix" $\left(u_{i}^{(k-1)}\right)_{i, k=1, \ldots, n}$, where $u_{i}$ is the solution of (27) determined by the initial conditions $u_{i}^{(k-1)}\left(t_{1}\right)=\delta_{i k}$. If $L^{(k)}, S^{*(k)}, X^{(k)}$ denote, respectively, the $k \times k$ submatrices of $L, S^{*}, X$ containing the element in the upper left corner, it follows from $L \in \tau, S^{*} \in \tau^{*}$ that $L^{(k)} S^{*(k)}=X^{(k)}$. The determinant of $X$ is the Wronskian $W_{k}=W\left(u_{1}, \cdots, u_{k}\right)$, and the determinant of a triangular matrix is the product of its diagonal terms. Since $L_{i i}=1$, we thus have $W_{k}=S_{11} S_{22} \cdots S_{k k}$, and therefore

$$
S_{k k}=W_{k} / W_{k-1} \text {. }
$$

From equation (31) we find, on the other hand, that $S_{k k}^{\prime}=\left(L_{k+1, k}-L_{k, k-1}\right) S_{k k}$. Combining this with (32), we obtain

$$
\frac{W_{k}^{\prime}}{W_{k}}-L_{k+1, k}=\frac{W_{k-1}^{\prime}}{W_{k-1}}-L_{k, k-1} .
$$

Since $W_{1}=u_{1}$ and, by (22), $L_{21}=u_{1}^{\prime}\left(u_{1}\right)^{-1}$, this shows that

$$
L_{k+1, k}=\frac{W_{k}^{\prime}}{W_{k}}, \quad k=1, \cdots, n-1 .
$$

Near $t_{1}$, the solution $u_{k}$ of (27) is of the form

$$
u_{k}=[(n-k) !]^{-1}\left(t-t_{1}\right)^{n-k}+O\left[\left(t-t_{1}\right)^{n}\right],
$$

and it is easy to see that this implies $W_{k}=O\left[\left(t-t_{1}\right)^{k(n-k)}\right]$. We may therefore conclude from (33) that

$$
L_{k+1, k}=\frac{k(n-k)}{t-t_{1}}+O(1)
$$

near $t_{1}$. The singular behavior of the other nontrivial elements of $L$ can be obtained from (34) if it is observed that, in accordance with the equations (29), the elements $L_{i k}, k<i-1$, can be computed from the elements $L_{k+1, k}$ by means 
of differentiations, multiplications and additions, and it is easily seen that $\lim _{t \rightarrow t_{1}^{\dagger}}\left(t-t_{1}\right)^{i-k} L_{i k}$ exists for all $k<i$. However, the knowledge of the singular behavior of the elements $L_{i k}, k<i-1$, is not necessary in order to characterize the solution $L$ of (28) in which we are interested. Because of the observation just made, $L$ can be obtained from the elements $L_{k+1, k}$ by means of elementary processes, and it is clear that $L$ is completely determined by the initial conditions $\lim _{t \rightarrow t^{+}}\left(t-t_{1}\right) L_{k+1, k}=k(n-k), k=1, \cdots, n-1$.

The following statement sums up our results.

THEOREM V. Let $L_{i k}, i=1, \cdots, n, k=1, \cdots, n-1$, be the (unique) solution of the Riccati system (29) determined by the conditions $\lim _{t \rightarrow t_{1}^{+}}\left(t-t_{1}\right) L_{k+1, k}=k(n-k), k=1, \cdots, n-1$. In order that the linear $n$th order equation (27) be disconjugate on the interval $\left[t_{1}, t_{2}\right)$ it is necessary and sufficient that the $L_{i k}$ be continuous on $\left(t_{1}, t_{2}\right)$.

In fact, our argument shows that it is sufficient to require the continuity of the $L_{k+1, k}, k=1, \cdots, n-1$. Since, according to (33), the continuity of these functions is equivalent to the conditions $W_{k} \neq 0, k=1, \cdots, n-1$, this provides a new proof of Pólya's well-known necessary and sufficient condition for disconjugacy [12].

\section{REFERENCES}

1. F. R. Gantmacher, The theory of matrices, GITTL, Moscow, 1953; English transl., Vol. 1, Chelsea, New York, 1959. MR 16, 438; 21 \#6372c.

2. M. Hanan, Oscillation criteria for third-order linear differential equations, Pacific J. Math. 11 (1961), 919-944. MR 26 \#2695.

3. G. H. Hardy, J. E. Littlewood and G. Pólya, Inequalities, 2nd ed., Cambridge Univ. Press, New York, 1952. MR 13, 727.

4. P. Hartman and A. Wintner, On disconjugate differential systems, Canad. J. Math 8(1956), 72-81. MR 17, 611.

5. W. J. Kim, Disconjugacy and disfocality of differential systems, J. Math Anal. Appl. 26 (1969), 9-19. MR 38 \#4759.

6. A. Lasota and C. Olech, An optimal solution of Nicoletti's boundary value problem, Ann. Polon. Math. 18 (1966), 131-139. MR 34 \#4580.

7. A. Ju. Levin, Some problems on the oscillation of solutions of linear differential equations, Dokl. Akad. Nauk SSSR 148 (1963), 512-515 = Soviet Math. Dokl. 4 (1963), 121-124. MR 26 \#3972.

8. Distribution of the zeros of solutions of a linear differential equation, Dokl. Akad. Nauk SSSR 156 (1964), 1281-1284 = Soviet Math. Dokl. 5 (1964), 818-821. MR 29 \#1378.

9. The non-oscillation of solutions of the equation $x^{(n)}+p_{1}(t) x^{(n-1)}+$ $\ldots+p_{n}(t) x=0$, Uspehi Mat. Nauk 24 (1969), no. 2 (146), 43-96 = Russian Math. Surveys 24 (1969), no. 2, 43-99. MR 40 \#7537.

10. Z. Nehari, Oscillation theorems for systems of linear differential equations, Trans. Amer. Math. Soc. 139 (1969), 339-347. MR 39 \#542. 
11. Z. Nehari, Nonoscillation and disconjugacy of systems of linear differential equations, J. Math. Anal. Appl 42 (1973), 237-254.

12. G. Pólya, On the mean-value theorem corresponding to a given homogeneous differential equation, Trans. Amer. Math. Soc. 24 (1922), 312-324.

13. B. Schwarz, Norm conditions for disconjugacy of complex differential systems, J. Math. Anal. Appl. 28 (1969), 553-568. MR 40 \#2963.

14. T. L. Sherman, Properties of solutions of $n$-th order linear differential equations, Pacific J. Math. 15 (1965), 1045-1060. MR 32 \#2654.

15. C. A. Swanson, Comparison and oscillation theory of linear differential equations, Academic Press, New York, 1968.

16. H. W. Turnbull and A. C. Aitken, An introduction to the theory of canonical matrices, Dover, New York, 1961. MR 23 \#A906.

DEPARTMENT OF MATHEMATICS, CARNEGIE-MELLON UNIVERSITY, PITTSBURGH, PENNSYLVANIA 15213 